\title{
Water-Formamide Mixtures: Topology of the Hydrogen-Bonded Network
}

\author{
Imre Bakó, ${ }^{1}$ Julianna Oláh, ${ }^{2}$ Anikó Lábas, ${ }^{2}$ Szabolcs Bálint, ${ }^{1}$ László Pusztai, ${ }^{3}$ Marie \\ Claire Bellissent Funel ${ }^{4}$ \\ ${ }^{1}$ Institute of Organic Chemistry Research Centre for Natural Sciences, Hungarian \\ Academy of Sciences, Magyar Tudósók körútja 2, P.O. Box 286, Budapest, Hungary \\ ${ }^{2}$ Department of Inorganic and Analytical Chemistry, Budapest University of Technology \\ and Economics, H-1111 Szent Gellért tér 4. Budapest, Hungary \\ ${ }^{3}$ Institute for Solid State Physics and Opics, Wigner RCP of the H.A.S. H-1121 Budapest, \\ Konkoly Thege út 29-33, Hungary \\ ${ }^{4}$ Laboratoire Leon Brillouin, CEA-SACLAY 91191 Gif sur Yvette, Cedex, France \\ Dedicated to prof. Vojko Vlachy
}

\begin{abstract}
A neutron diffraction measurements and molecular dynamics simulations have been performed to study the hydrogen bonded structure of water-formamide mixtures. We showed that the calculated and measured total neutron diffraction radial distribution function agreed very well. After evaluation of this function, the hydrogen-bonded structure of mixtures with various compositions has been studied, and the clustering properties and the topology of the hydrogen-bonded network were investigated. The results show that these mixtures exhibit an extended range structure in solution. In waterformamide mixtures the average number of hydrogen bonded neighbors (water, formamide) and the distribution of the number of H-bonded neighbors do not change significantly as a function of the formamide mole fraction. Molecules are shown to form percolated networks at each concentration. The composition of cyclic entities in these systems is very close to being ideal; that is, these systems are microscopically homogeneous.
\end{abstract}

\section{Introduction}

It is known that hydrogen bonds (H-bonds) play important roles in a wide variety of complex materials, such as biological macromolecules or supramolecular systems (see, e.g. Refs. [1-12]. Amides are the simplest organic molecules that can be models of peptide bonds. Interactions between peptide groups play an essential role in shaping the structure and properties of biological systems, such as proteins and nucleic acids, as well as the behavior of many solvent systems. Hydration properties of amide groups during the folding process are leading factors for determining the secondary and ternary structure of proteins.

Formamide, $\mathrm{H}-\mathrm{CO}-\mathrm{NH}_{2}$, is the simplest molecule containing an amide group that can represent a simplistic model of the peptide bond. The intermolecular structure of liquid formamide has been the subject of several theoretical and experimental studies (see Refs. [13-21]). It has been shown that a three-dimensional hydrogen-bonded network exists in liquid formamide, similar to that found in liquid water [14,17.21]. Additionally, 
it was suggested that the primitive ring distribution in liquid formamide and water are significantly different [21]; this statement was corroborated by static dielectric studies [13].

Interactions between water and formamide molecules have received a great deal of attention, too (see, e.g., Refs. [22-30). One of the main conclusions of these studies was that, based on the structural parameters, these systems can be regarded as a prototype of an ideal mixture [22,24]. In contrast, Perera et al. revealed structural microheterogenities in aqueous amides [27]. Ladanyi et al revealed that the excess internal energy of mixing is a small positive value calculated from molecular dynamics simulations, and it agrees very well with the experimentally determined heat of mixing. [31]

The structure of these systems at the molecular level can be described as a complex network. which, in turn, may be characterized by its topological properties. Recently, we described the topology of hydrogen bonded aggregations in water [32] and pure liquid formamide [21], and we were able to detect a significant amount of structural microheterogenities in water/methanol mixtures [32].

The aim of the present study was to get new insight into the microscopic properties of formamide-water mixtures by characterizing their topological properties. First we carried out new neutron diffraction experiments on deuterated formamide-water mixtures and used it to evaluate the performance of the two interatomic potential models available for formamide in the literature [33-35]. Next, we applied graph theory adjusted for the study of hydrogen bonded networks in order to get a description of the topological properties of the H-bonded network in liquid water/formamide mixtures.

\section{Wide angle neutron scattering}

Neutron diffraction experiments on fully deuterated liquid samples, with mole fractions of $\mathrm{DCOND}_{2} \mathrm{x}_{\mathrm{fa}}=0.1,0.3,0.5,0.7$ in $\mathrm{D}_{2} \mathrm{O}$, have been conducted on the hot-source diffractometer 7C2 of the Laboratoire León Brillouin (CEA Saclay, France). The liquids were placed in a vanadium container with diameters of $6 \mathrm{~mm}$ and wall thickness of 0.1 $\mathrm{mm}$. The incident neutron wavelength was $0.70 \AA$. For standard correction and normalization procedures, additional runs (vanadium rod, cadmium rod, empty container and background) were also performed. Raw diffraction data were corrected for background, container- and sample absorption, and multiple scattering; intensities then were normalized by making use of scattering data on the vanadium rod sample. A more detailed description of the correction procedure can be found in Ref. [36-38]. Experimental total scattering intensities are given in Figure 1, while neutron weighted total structure factors are provided in Figure 2a (numerical data are available upon request from the authors).

The conversion of the observed interference function, $I(Q)$, to an $r$-space representation (i.e., the pair correlation function) is normally conducted by numerical Fourier transformation. This process leads to various inaccuracies arising from statistical fluctuations, limited $Q$-range and possible systematic errors in the original data sets. Here we apply a different approach to solve this problem, developed by Pusztai and McGreevy [39], called MCGR, which is an acronym for 'Monte Carlo treatment of the $\mathrm{g}(\mathrm{r})$ ' function. This procedure uses an inverse approach in which the pair correlation function (total or partial) is generated numerically, and modified by a random process until its 
inverse Fourier transform $I(Q)$ agrees with the experimentally measured $I_{o b s}(Q)$ within its errors. A more detailed description of the procedure is given in Ref. [40] and is also available from the World Wide Web [41].

The comparison of the measured total neutron structure factors for formamidewater mixture with those resulted at the end of MCGR procedure is given in Figure 2a; agreement between MCGR model and experiment is excellent. The total radial distribution function and the corresponding water and formamide intramolecular contributions are shown in Figure $2 b$.

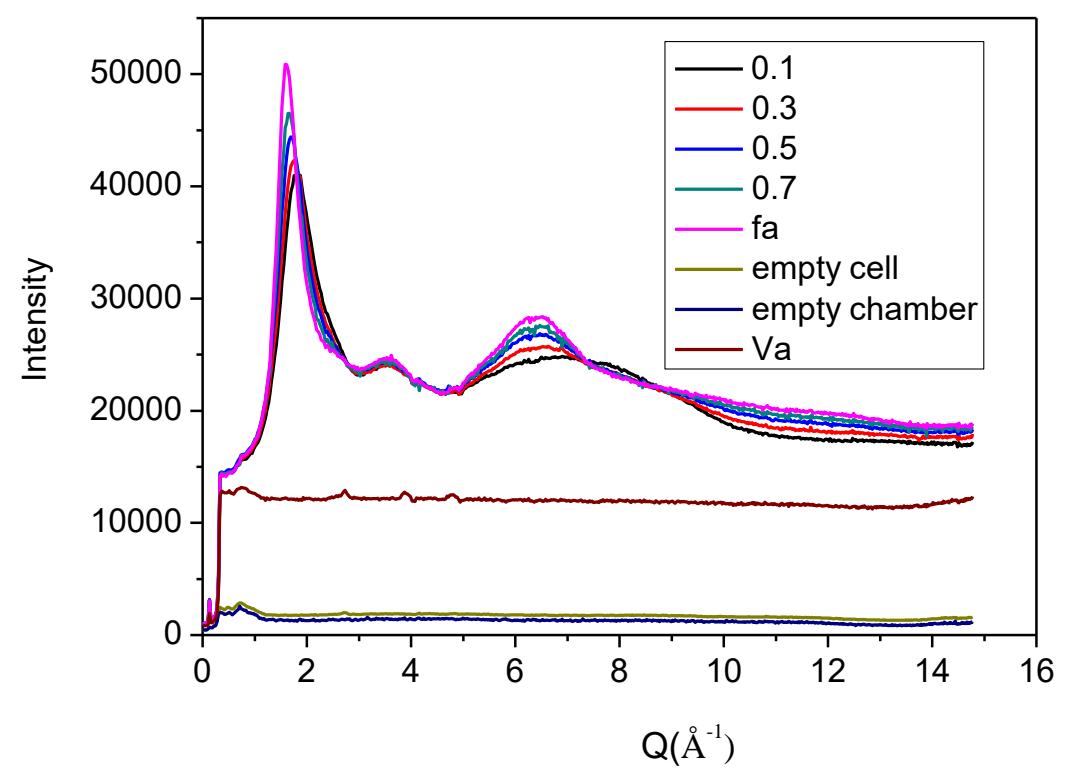

Figure 1 Experimental total scattering intensities for different formamide mole fraction values. Data are also given for the empty cell, empty chamber and for the vanadium bar. 


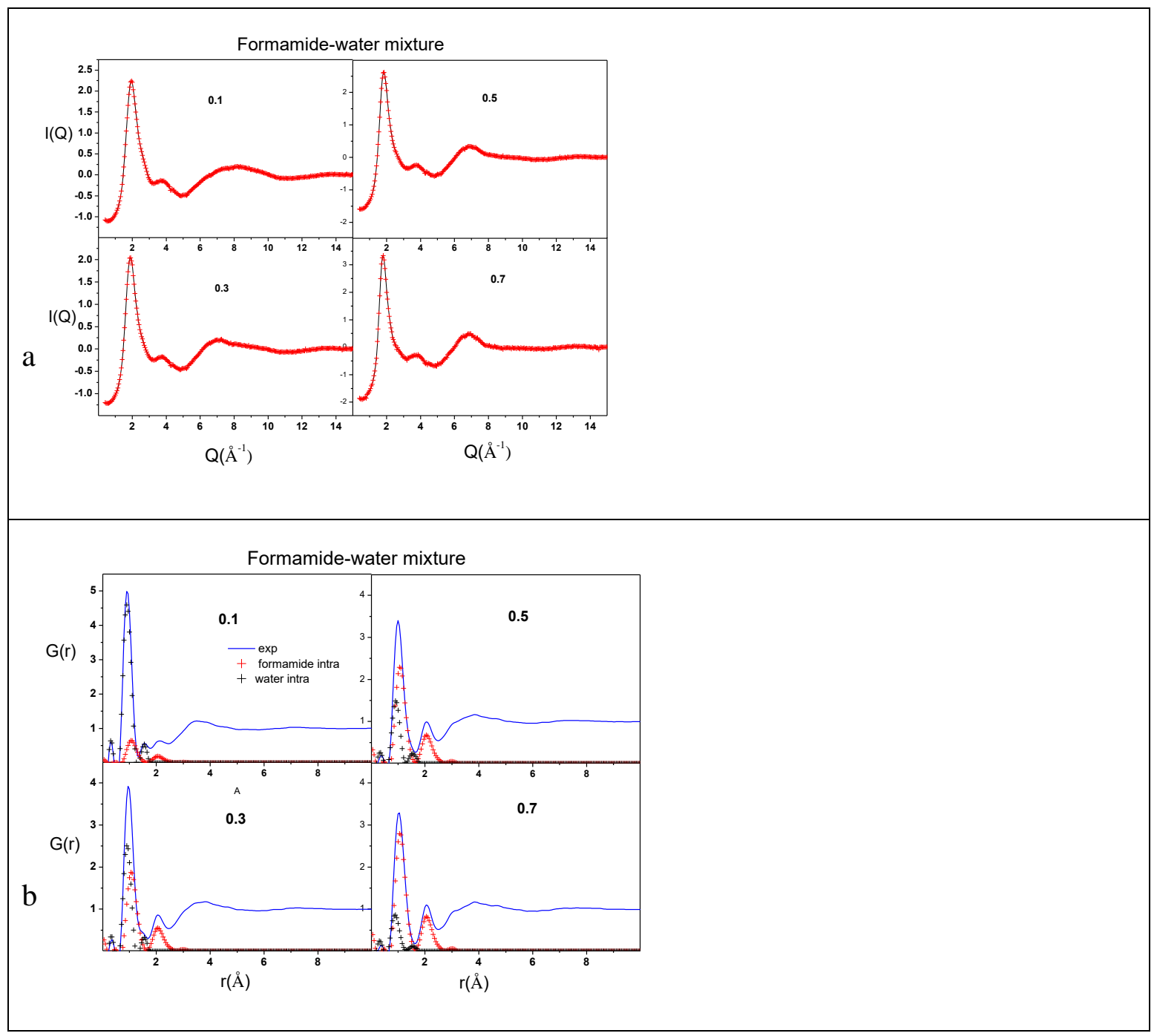

Figure 2a. Comparison of the corrected total neutron structure functions ( I(Q)) (dots) of liquid $\mathrm{DCOND}_{2} / \mathrm{D}_{2} \mathrm{O}$ mixture $x_{\mathrm{fa}}=0.10,0.30,0.50,0.7$ with those obtained after the MCGR procedure (full line).

Figure 2b. Total experimental neutron pair correlation function of liquid-liquid $\mathrm{DCOND}_{2} / \mathrm{D}_{2} \mathrm{O}$ mixture $x_{\mathrm{fa}}=0.10,0.30,0.50,0$. (full line) and Fourier transform of intramolecular contributions $I_{\mathrm{M}}(Q)$ of water and formamide molecule to the total structure function (cross).

\section{Computer simulation details}

We have performed classical molecular dynamics simulations in the NVT ensemble using the Nose-Hoover thermostat at an average temperature of $298 \mathrm{~K}$ on liquid formamide-water mixtures at formamide mole fractions of $x_{\mathrm{fa}}=0.00,0.10,0.30,0.50$, $0.7,0.90,1.00$. The simulations were performed with the six-site (i.e., 'all atom' type) OPLS [33,34] and Cordeiro [35] potentials for formamide; in both cases, SPC/E [42] 
water was the solvent. Lorentz-Berthelot combining rules were applied to calculate the mixed potential terms. In all simulations the periodic cube contained 2048 molecules; side lengths of the cubes corresponded to the experimental densities) [43]. The corresponding box lengths are given in the Supplementary material. The timestep in every simulation run was 1 fs.

Short-ranged intermolecular potential interactions were truncated at half of the corresponding box-size. Periodic boundary conditions were employed and the Ewald summation was used to handle the long-range Coulomb interactions.

All simulations were performed using the DLPOLY 2.16 software [44]. After performing a 1ns-long equilibrations, the trajectories were saved in every 1000 steps, and $10 \mathrm{~ns}$ of simulation times (altogether $10^{4}$ structures for each system) were used for calculating the quantities of interest.

Average hydrogen bond number, $\mathrm{N}_{\mathrm{HB}}$, was calculated by averaging the number of hydrogen bonds over the trajectory and over all molecules

\section{Comparison between neutron diffraction and molecular dynamics simulations: Composite intermolecular radial distribution functions}

We have performed neutron diffraction studies on liquid formamide-water mixtures at room temperature and determined the total RDFs (G(r) (see above), that can be used to check the abilities of the interatomic potentials employed in our computer simulations. The total (or 'composite') structure factors are the weighted sum of the partial structure factors

$I(Q)=\sum w_{\alpha \beta} I_{\alpha \beta}(Q)$

where

$w_{\alpha \beta}=\frac{\left(2-\delta_{\alpha \beta}\right) x_{\alpha} x_{\beta} b_{\alpha} b_{\beta}}{\left(\sum x_{\alpha} b_{\alpha}\right)^{2}}$

and $\mathrm{b}_{\alpha}$ is the neutron scattering length of species $\alpha\left(\mathrm{Ow}, \mathrm{Hw}, \mathrm{C}_{\mathrm{fa}}, \mathrm{O}_{\mathrm{fa}} \ldots\right)$ and $I(Q)$ is the total structure factor. The total (or 'composite') radial distribution, $G(r)$, is the Fourier transform of $I(Q)$. At this point we have already subtracted the intramolecular scattering contributions arising from individual water and formamide molecules [21]

The $G(r)$ from experiment and simulation are compared in Figure 3; the agreement between the experiment and simulation is, generally speaking, good for both potential models. Interestingly, it is the water-rich side, at formamide mole fractions of 0.1 and 0.3 , where any discernible disagreement could be spotted visually, in the vicinity of the main maximum of $G(r)$. This fact may be an indication that while both formamide potentials work reasonably well for pure formamide and for formamide-rich mixtures, some attention is needed when interactions between formamide and water molecules start to dominate the scene (as opposed to the dominantly formamide-formamide neighbourhood). 


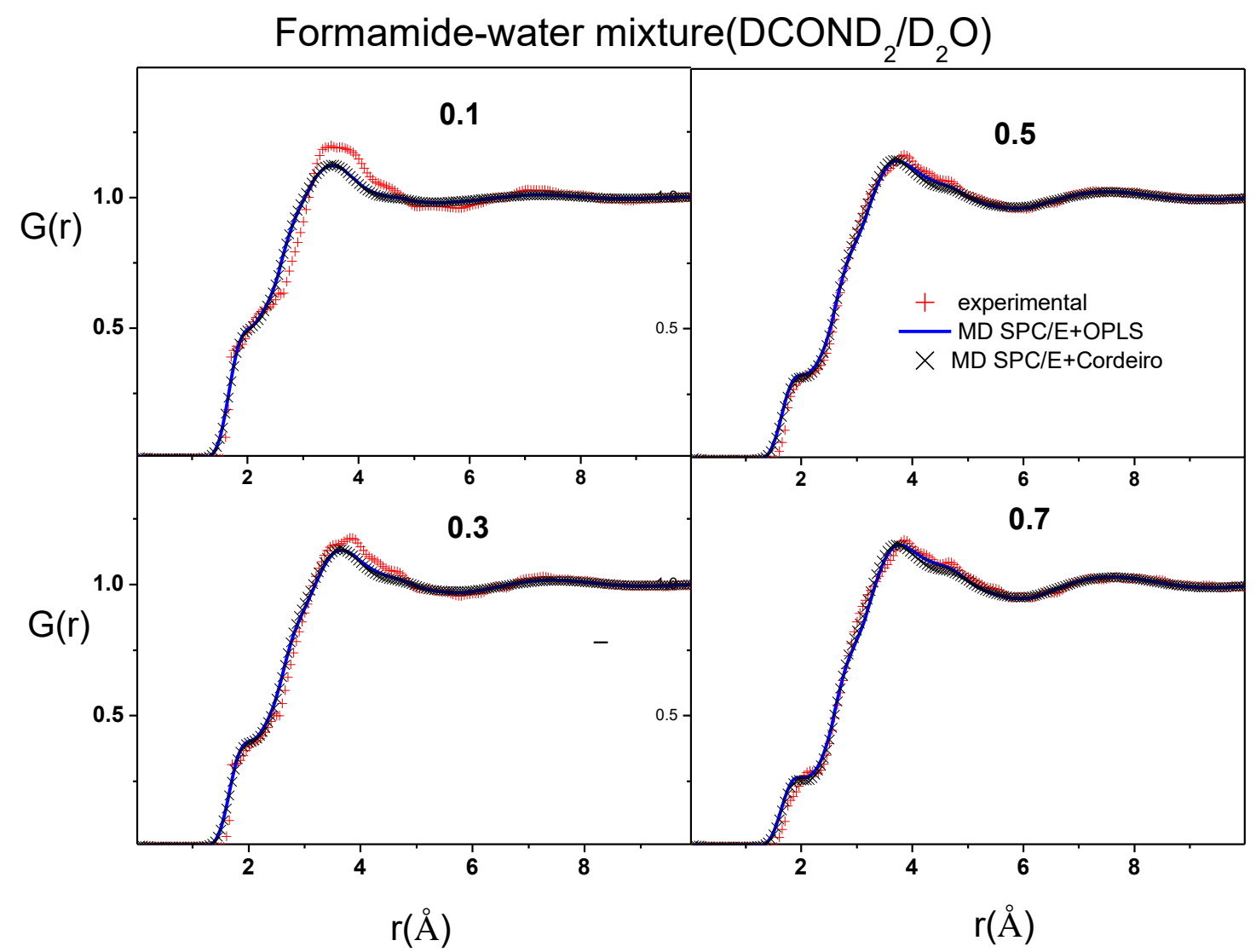

Figure 3. Comparison of intermolecular radial distribution functions (of liquid $\mathrm{DCOND}_{2} / \mathrm{D}_{2} \mathrm{O}$ mixture $\left.x_{\mathrm{fa}}=0.10,0.30,0.50,0.7\right)$ obtained from wide-angle neutron diffraction experiment(cross) and molecular dynamics simulation using Cordeiro [35] and OPLS model $[33,34]$ for formamide .

\section{Hydrogen bond analyses}

As the radial distribution functions obtained from neutron-diffraction data and from simulation showed very good agreement suggesting that modeling gives a realistic description of liquid formamide-water mixtures, we turned our attention towards the properties of the hydrogen bond network in these mixtures. Hydrogen bond statistics can give us topological information about the average local hydrogen bonded network structure in a (H-bonded) liquid. Appropriate definitions of hydrogen bond, with one molecule in the centre of the coordinate system, are usually based on energetic and/or geometric criteria (see, e.g., Refs. [45-47]). We employed two different H-bond definitions here. (1) geometric or distance-angle definition: we considered two molecules to be $\mathrm{H}$-bonded if the O...H distance was shorter than $2.7 \AA$ and $2.5 \AA$ for $\mathrm{N}-\mathrm{H} . . \mathrm{O}$ and $\mathrm{O}-\mathrm{H} . . \mathrm{O}$ neighborhoods, respectively, and the $\mathrm{O} \ldots \mathrm{O}-\mathrm{H}$ or $\mathrm{O} \ldots \mathrm{N}-\mathrm{H}$ angle was smaller than $30^{\circ}$. Here, it is worth noting that the carbonyl group in formamide can only act as a hydrogen bond acceptor, its amide group as hydrogen bond donor (as the lone pair of the 
amide nitrogen is significantly delocalized towards the carbonyl group greatly reducing its ability to accept a hydrogen bond) and water can be both a hydrogen bond donor or acceptor. (2) Distance-energy definition: the distance cutoff was the same as above and the energy threshold was $-12.4 \mathrm{~kJ} / \mathrm{mol}$ [21], which is the position of the minimum of the pair-interaction energy distribution for formamide-formamide, formamide-water and water-water molecule pairs. As usage of both hydrogen-bond definitions led to the same conclusions only results obtained from the Cordeiro/SPC/E model using the geometric definition will be presented. We would like to mention usage of stricter criteria: either a smaller angle $\left(20^{\circ}\right)$ in the case of the geometric criteria or a smaller interaction energy threshold $(4.18 \mathrm{~kJ} / \mathrm{mol})$ for the energetic criteria, leads to very similar results and do not change the conclusions of our study at all.

In Figure 4a. we depicted the average hydrogen bond number as a function of the formamide mole fraction (all-all). This number has also been decomposed to various terms to get information on the formamide-formamide (fa-fa), formamide-water (fa-wa), water-water (wa-wa) and water-formamide (wa-fa) subsystems. Please note that the tottot value, is not a simple sum, but a weighted sum of the formamide-all (fa-all) and water-all (wa-all) contributions. The number of $\mathrm{H}$-bonds between the formamide and water pair is changed almost linearly. It is also clear that the average H-bonded environment around formamide and water molecules does not change significantly with formamide mole fraction. We can make the same conclusion from Figure $4 \mathrm{~b}$. where we show the distribution of the number of hydrogen bonds for all molecule pairs (without distinguishing molecule types). This finding is very similar to that of Elola et al. [22,23] for an OPLS/SPC/E potential combination who used a somewhat different H-bond definition. 


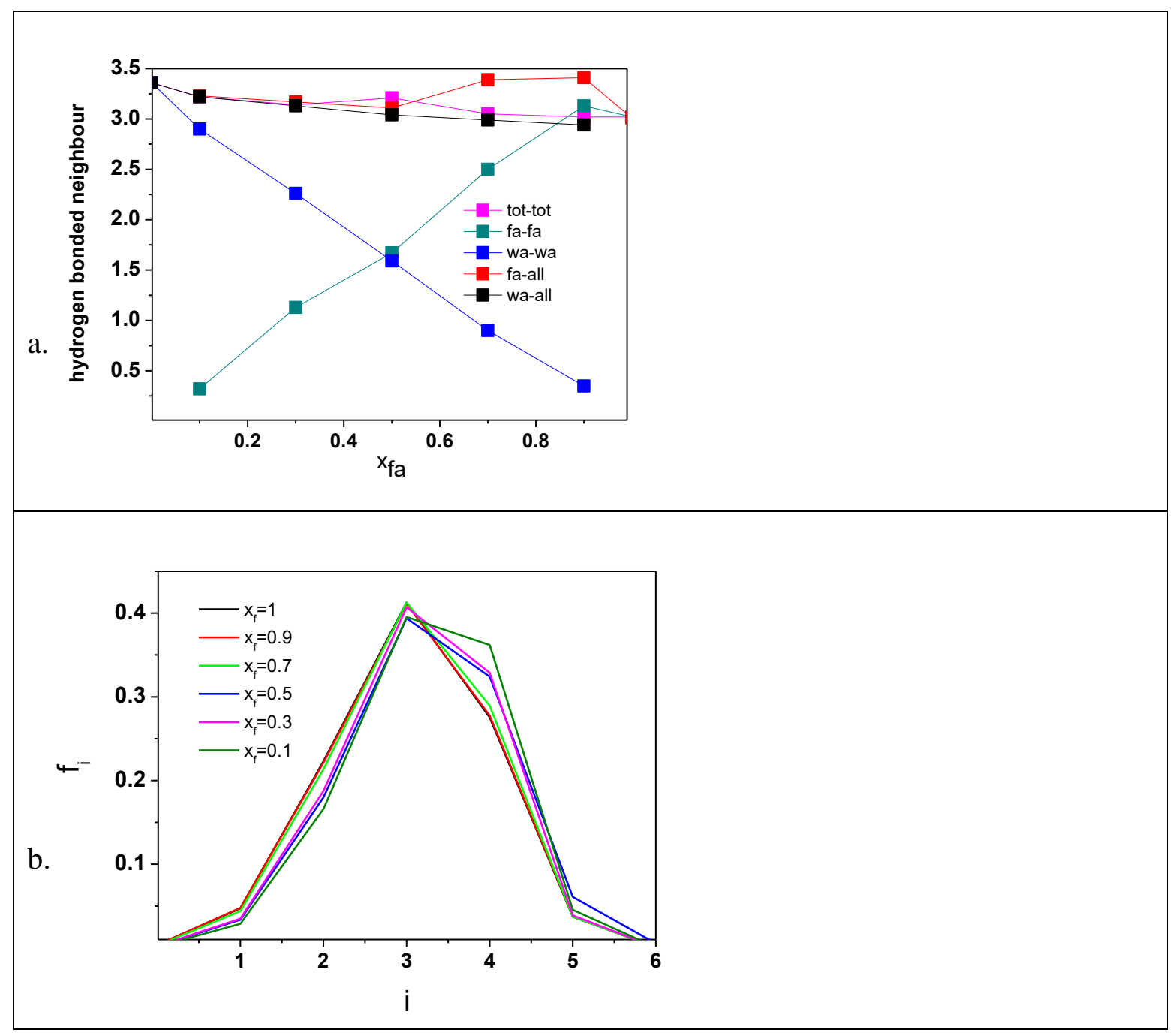

Figure 4.a Average hydrogen bond number $\left(\mathrm{N}_{\mathrm{HB}}\right)$ as a function of formamide mole fraction $\left(\mathrm{x}_{\mathrm{fa}}\right)$ for all of the molecule and for different subsystems like water-water, formamide-formamide, water-all, and formamide-all. (Geometric H-bond definition was applied)

Figure $4 \mathrm{~b}$. Fraction of hydrogen bonded neighbour molecules (water or formamide) as a function of $\mathrm{n}=0 \cdots 5$ hydrogen bonds at various concentrations

There are several indications for a random component arrangement in this system (mainly ideal mixing properties.) We calculated a parameter that can be applied as a descriptor for inhomogeneity in the particle configurations. We define the following localisation parameter [24]:

$x_{l o c}=\frac{n_{\alpha \beta}}{\left(n_{\alpha \beta}+n_{\alpha \alpha}\right)}$ 
where $n_{\alpha \beta}$ is the average number of $H$-bonds for the $\alpha-\beta$ pair ( $\alpha$ and $\beta$ can be either a formamide or a water molecule). As shown in Figure 5, this parameter depends on the mole fraction of formamide nearly linearly for formamide-water and water-formamide pairs. It is therefore proven that at this level we can take our solutions as prototypes of an ideal mixture.

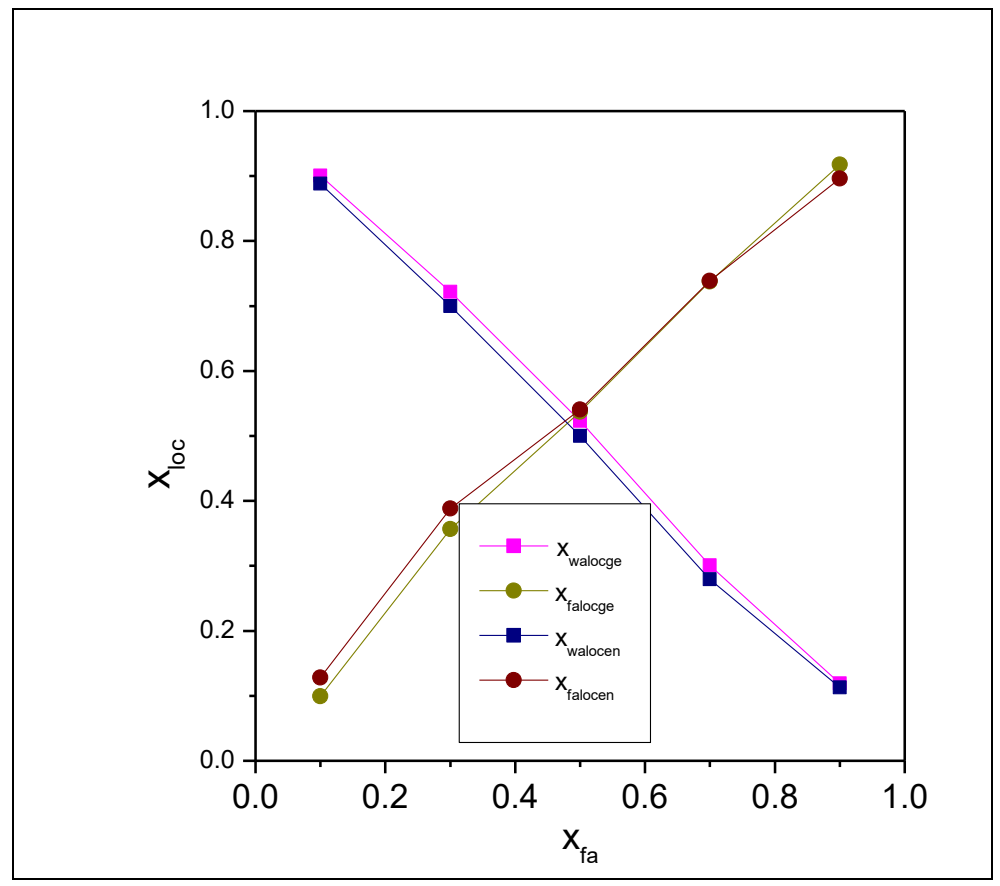

Figure 5. The localization parameter for water and formamide with geometric(ge) and energetic(en) H-bond definition as a function of formamide mole fraction

\section{Clustering and percolation}

Two molecules are participate in the same cluster, according to the definitions introduced by Geiger et al. [48-50], if they are connected by a chain of hydrogen bonds. It has been already shown that both water and formamide molecules form a three dimensional percolating hydrogen bonded network in their pure liquid phase [30,32]. Percolation can be monitored by the comparison of the calculated cluster size distribution function of the present system with that obtained for random percolation on a $3 \mathrm{D}$ cubic lattice, $\mathrm{P}\left(\mathrm{n}_{\mathrm{c}}\right)=\mathrm{n}_{\mathrm{c}}{ }^{-2.19}[51,52]$. In systems that are below the percolation limit the $\mathrm{P}\left(\mathrm{n}_{\mathrm{c}}\right)$ distribution deviates significantly down from the equation for random percolation already at small cluster sizes. On the other hand, in percolating systems the $\mathrm{P}\left(\mathrm{n}_{\mathrm{c}}\right)$ distribution strongly exceeds the above defined function at large cluster size values and drops below it at intermediate cluster sizes.

In our case the total (i.e., when not distinguishing between molecule types) $\mathrm{H}$ bonded network is percolated at each concentration, which is not too surprising due to the 
fact that the average H-bonds per molecule value is about 3.0 or more. Figure 6 presents the hydrogen-bonded cluster size distribution in formamide-water mixtures at various concentrations for two different subsystems, namely the water-water and formamideformamide (sub)networks. It can be observed that in the water-water subsystem, up to $x_{\mathrm{fa}}$ $=0.3$, water molecules are percolated throughout the system as the formamide concentration is increased. As the concentration of formamide increases, the formamideformamide clusters also build up a percolated network. The mole fraction of formamide molecules where this subsystem becomes a percolated network is slightly larger than $x_{\mathrm{fa}}=$ 0.5 . We also observe that if the mole fraction of water or formamide is less than or equal to $x_{f a}=0.3$ or $x_{w}=0.3$, the minor component will form small $(<30)$, isolated formamide or water clusters, respectively. However, at mole fractions $x_{f a}=0.5$ both subsystems are very close to the percolation limit (see positions of the blue lines and the dotted lines representing the percolation limit in Figure 6.). Typical hydrogen bond network topologies for all the molecules, as well as for the water-water and formamide-formamide subsystems, at the composition of $x_{f a}=0.5$ are shown in Figure 7 


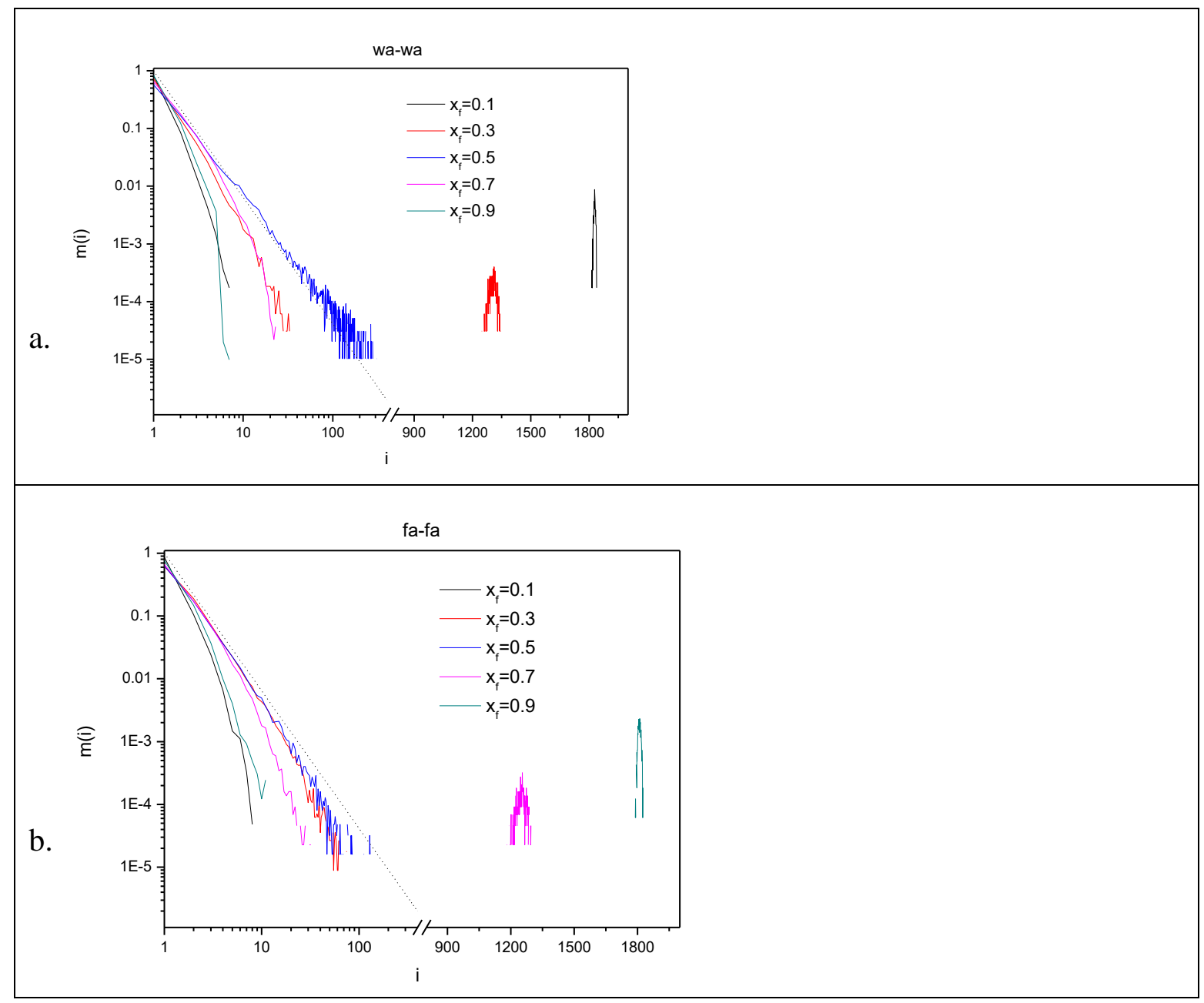

Figure 6. Cluster size distribution of water-water (a) and formamide-formamide subsystems (b) in the water-formamide mixture at various concentrations. Dots represent the limit for a random percolation network $\mathrm{P}\left(\mathrm{n}_{\mathrm{c}}\right)=\mathrm{n}_{\mathrm{c}}{ }^{-2.19}$. 


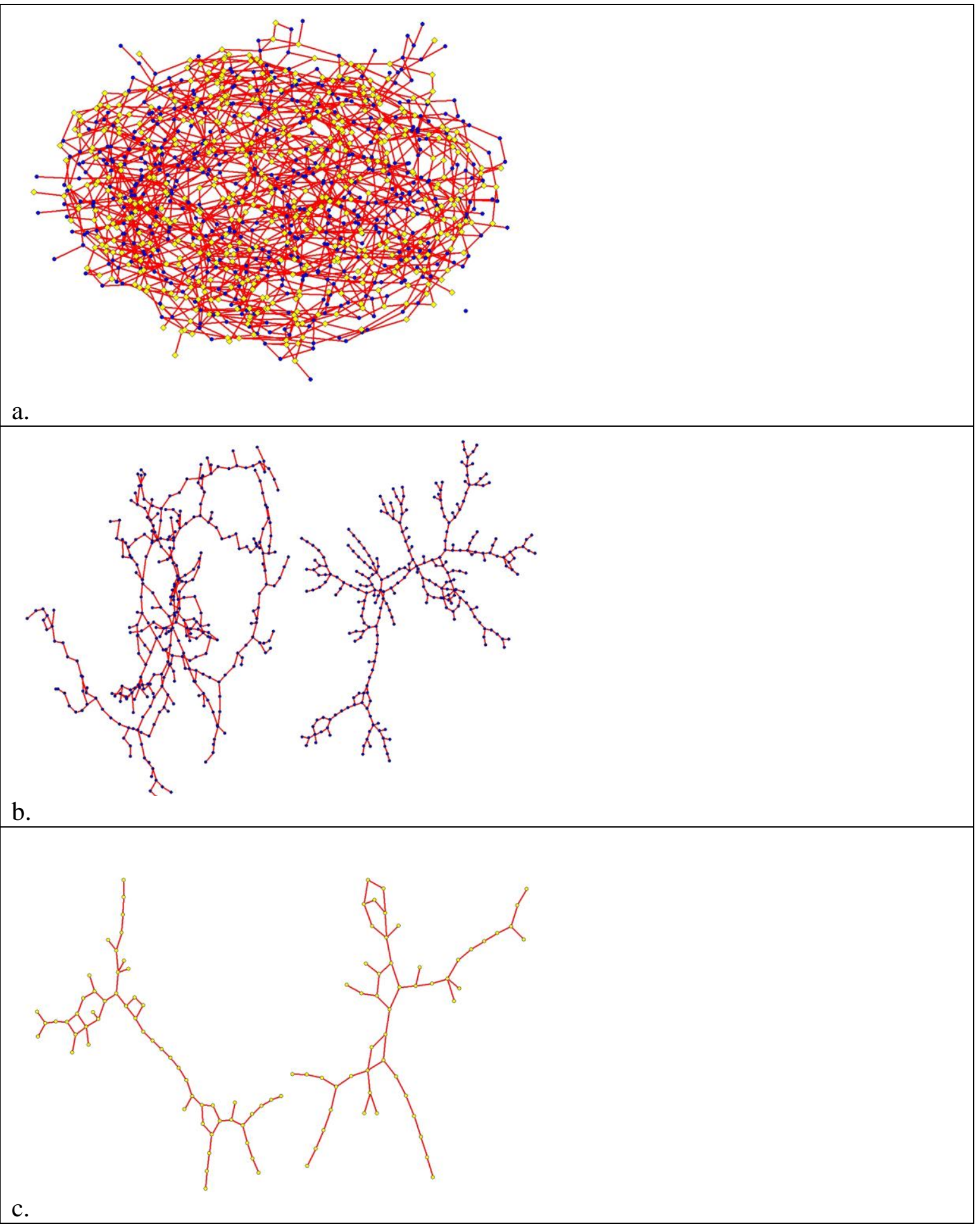

Figure 7. Hydrogen bonded network topology of (a) a typical configuration in the water-formamide mixture (b) of its formamide-formamide subsystem (c) of its waterwater subsystem at $\mathrm{x}_{\mathrm{fa}}=0.5$ mole fraction (yellow circles: water, blue circles: formamide), The figure was drawn using the Pajek code [53-55] 
Clearly, these systems contain several cyclic entities. Molecules participate in a given cyclic entity if there is a minimum length path consisting of a series of hydrogen bonds thorugh which one can get back to the original molecules. We can define the size of a cyclic entity as the shortest path through the hydrogen bonds. To estimate the distribution of such cyclic entities, the ring search algorithm developed by Chiaia et al.[56] was used in this work; the same method has already been used in the investigation of the hydrogen-bonded network topology in liquid formamide and in water-methanol mixtures, too. The average number of cyclic entities is denoted by $N_{c}$; Figure 8 shows the normalized number of cyclic entities $\left(\mathrm{N}_{\mathrm{c}} / \mathrm{N}\right.$, where $\mathrm{N}$ is the total number of molecules) as a function of the formamide mole fraction $\left(x_{\mathrm{fa}}\right)$. One can observe that the number of cyclic entities does not change significantly with increasing formamide concentration. The ring size distribution is presented in Figure 9 as a function of cycle size $\left(\mathrm{n}_{\mathrm{c}}\right)$. In the case of pure water the ring-size distribution has a well-defined maximum around 6 and 7 , whereas for pure formamide, the maximum can be found at 11. This difference between the structure of these two liquids was already pointed out by dielectric spectroscopy [13]. The shape of the ring size distribution changes continuously as a function of the formamide mole fraction.

We have already used a topological parameter for defining the microscopical heterogentity in water-methanol mixtures. [32] We calculated the total number of water and methanol molecules that participate in a cyclic entity with a given size $\left(\mathrm{m}_{\mathrm{c}}\right.$ denotes the size of the cycle). If the system is microhomogenous in this sense the ratio of these two numbers calculated for the two components should be very close to the molar ratio of the components for each $\mathrm{m}_{\mathrm{c}}$. In contrast, if the system is microheterogenous (e.g. watermethanol mixtures [32]), the ratio is very far from the molar ratio.

It was found that water molecules are likely to be part of cyclic entities in an extended 3D network. This difference in methanol-water mixture can be clearly explained by the different $\mathrm{H}$-bonded network topology in pure water (3D H-bond network) and methanol (short branched chains). Figure 10 shows the ratio of formamide and water molecules that belong to $m_{c}$ different cycles.

In methanol-water mixtures we had previously found that more methanol molecules appear in non-cyclic entities, while more water molecules are connected to rings, than it would follow from the composition. This behaviour could be interpreted as a signature of microscopic configurational inhomogenities in water-methanol mixtures; we therefore made use of this finding for determining possible microscopic configurational inhomogenities in the present mixtures. On the basis of this analysis it is revealed that the composition of cyclic entities in formamide-water mixtures is very close to the ideal one (i.e., to that of the composition of the entire system), thus a microscopic homogeneity was found in the present solutions. 


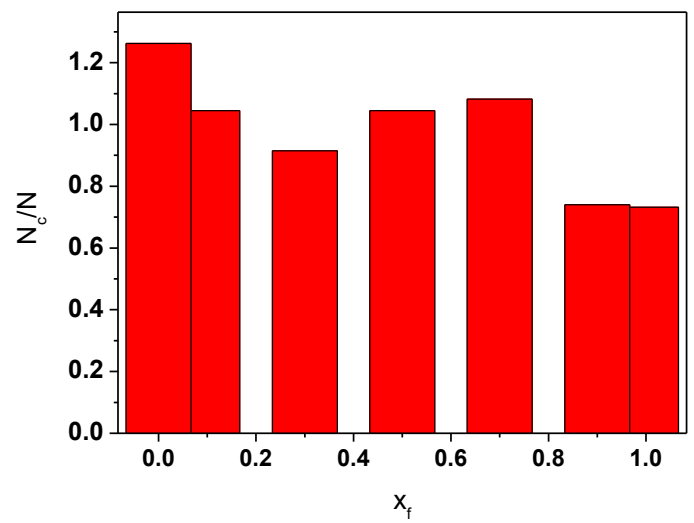

Figure 8 . Number of cyclic entities compared to the total number of molecules in the simulation box as a function of formamide mole fraction.

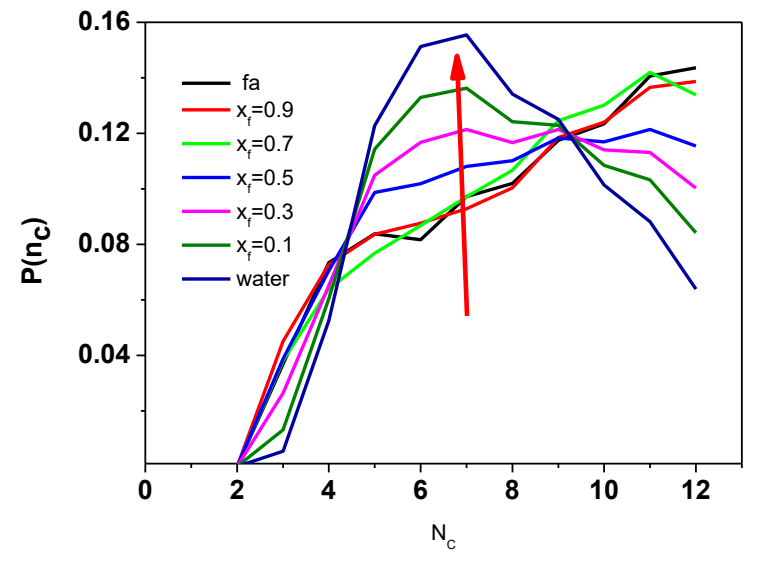

Figure 9. Size distribution of cyclic entities as a function of $\mathrm{n}_{\mathrm{c}}$, number of molecules forming the cycle. 


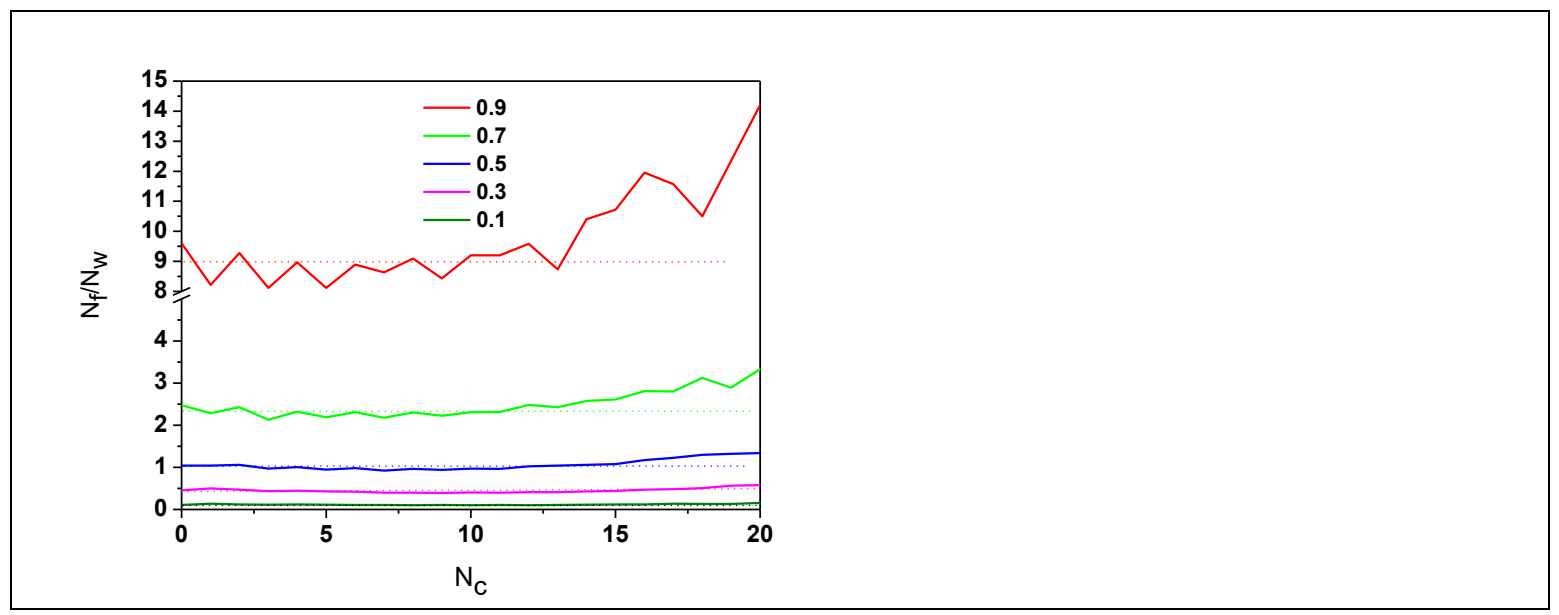

Figure 10 Ratio of formamide and water molecules belonging to $m_{c}$ different cycles at various formamide mole fractions represented by different colours $\left(\mathrm{x}_{\mathrm{fa}}\right)$.

Conclusion

We have studied the structure of water-formamide mixtures using neutron-diffraction and molecular dynamics simulations. At all studied formamide concentrations we found a very good agreement between measured total radial distribution functions and those obtained from molecular dynamics simulations. calculated, which gave a reliable basis for further investigation of the microscopic structure and of the hydrogen bond network structure of these mixtures. In water-formamide mixtures the average hydrogen bonded neighbour number of molecules (water, formamide) and the distribution of H-bonded neighbour does not change significantly as a function of formamide mole fraction. In these mixtures the molecules form percolated network in all concentration. On the basis of the topological analysis reveals, that the composition of cyclic entities in these system are very close to the ideal, thus so we find a microscopic homogenity in this system.

Acknowledgements

A. L. thanks the financial support for the Richter Gedeon Talentum Foundation. J.O. was supported by the Bolyai János Research Scholarship and by NKFIH Grant No. 115503. LP is grateful for the financial support received from a grant of the National Innovation and Development Agency (Hungary), No. SNN 116198. I.B and J.O acknowledge the partial financial support of the Hungarian Scientific Research Fund (grant OTKA K108721). 


\section{References}

1. B. Bagchi, Water in Biological and Chemical Processes: From Structure and Dynamics to Function (Cambridge University Press, 2014).

2. M. Chaplin, "Water Structure and Science" London South Bank University http://www.lsbu.ac.uk/water/ (accessed 14 January, 2016).

3. D.Eisenberg, ; W.Kauzmann, W. The Structure and Properties of Water; Oxford University Press: Oxford, 2005.

4.F. Franks,. Water, a Matrix of Life (2nd ed.); Royal Society of Chemistry: Cambridge, 2000.

5. R. Ludwig, Water: From Clusters to the Bulk. Angew. Chem. Int. Ed. 40, 1808 (2001)

6. S J. Grabowski What Is the Covalency of Hydrogen Bonding Chem. Rev. 111, 2597 (2011)

7.G. A. Jeffrey, W.; Saenger, Hydrogen Bonding in Biological Structures; SpringerVerlag: Berlin, 1991.

8 G.A. Jeffrey, . An Introduction to Hydrogen Bonding; Oxford University Press: New York, 1997.

9 G.R. Desiraju,T..; Steiner, The Weak Hydrogen Bond in Structural Chemistry and Biology; Oxford University Press Inc.: New York, 1999.

10 S. J. Grabowski Hydrogen Bonding - New Insights;., Ed. In Series

Challenges and Advances in Computational Chemistry and Physics; Leszczynski,

J., Ed.; Springer: New York, 2006.

11 T. Steiner The Hydrogen Bond in the Solid State Angew. Chem. Int. Ed. 41 ,48 (2002)

12. S. Scheiner, Hydrogen Bonding. A Theoretical Perspective, Oxford

University Press, Oxford, 1997.

13. J. Jadzyn and J. Swiergiel On similarity of hydrogen-bonded networks in liquid formamide and water as revealed in the static dielectric studies Phys. Chem. Chem. Phys., 14, 3170-3175, (2012)

14. E. Tsuchida Ab initio molecular-dynamics study of liquid formamide J. Chem. Phys.121 4740 (2004)

15. S. N. ,N. Bosio X-ray scattering study of amorphous formamide J. Chem. Phys. 2297 108 (1998)

16. R. Ludwig, F. Weinhold, and T. C. Farrara Experimental and theoretical studies of hydrogen bonding in neat, liquid formamide 5118102 (1995)

17. T. Radnai T. Megyes, I. Bakó, T. Kosztolányi, G. Pálinkás, H. Ohtaki Structure and dynamics of liquid formamide at high pressure and high temperature: comparison of $\mathrm{X}$ ray diffraction and molecular dynamics results Journal of Molecular Liquids 110 ,123 (2004)

18. E. Kálmán, I. Serke, G. Pálinkás, M.D. Zeidler, F. J. Wiesmann, H, Bertagnolli, P. Chieux Z. Naturforsch 38a, 231,(1983)

19. J. Richardi, H. Krienke, P.H. Fries Chem. Phys. Letters 273,115, (1997)

20. F. J. Wiesmann, M.D. Zeidler, H. Bertagnolli, P. Chieux Mol. Phys. 57, 275, (1986)

21. I. Bakó, T. Megyes, Sz. Bálint, V. Chihaia, M.-C. Bellissent-Funel, H. Krienke, A.

Kopf, and S.-Hyuck Suh Hydrogen bonded network properties in liquid formamide J.

Chem. Phys 132, 014506 (2010) 
22. M. Dolores Elola and Branka M. Ladanyi "Computational study of structural and dynamical properties of formamide-water mixtures" J. Chem. Phys. 125, 184506_(2006) 23. M. Dolores Elola and Branka M. Ladanyi "Intermolecular polarizability dynamics of aqueous formamide liquid mixtures studied by molecular dynamics simulations" J. Chem. Phys. 126, 084504 (2007)

24. Yurii P. Puhovski and Bernd M. Rode Molecular dynamics simulations of aqueous formamide solution. II. Dynamics of solvent molecules” J. Chem. Phys. 1022920 (1995) 25. Yurii P. Puhovski and Bernd M. Rode Molecular Dynamics Simulations of Aqueous Formamide Solution. 1. Structure of Binary Mixtures J. Phys. Chem. 1995,99, 1566-1576 26. M.A.M. Cordeiro, W.P. Santana, R. Cusinato, J.M.M. Cordeiro Monte carlo investigations of intermolecular interactions in water-amide mixtures J. Mol. Structure THEOCHEM 759 159-164 (2006)

27. L. Zoranić, R. Mazighi, F. Sokolić, and A. Perera Concentration fluctuations and microheterogeneity in aqueous amide mixtures J. Chem. Phys. 130, 124315, (2009) 28. R.J. Sengwa. V.A Khatri and S. Sankhla Static Dielectric Constant, Excess Dielectric Properties, and Kirkwood Correlation Factor of Water-Amides and Water-Amines Binary Mixtures Proc. Indian Nat. Sci. Acad 74 67-71 (2008)

29. S. Bishwas, B.S. Mallik Aqueous solvation of an amide molecule from first principle simulations:structure, hydrogen bond dynamics and spectral signature J. Mol. Liq. 2015 212 941-946 (2015)

30.S. Tolosa , A. Hidalgo, J.A. Sans Thermodynamic, structural, and dynamic study of the N-H.. O-C hydrogen bond association in aqueous Chemical Physics 55,73, (2000) 31.

32 I. Bakó, T. Megyes, Sz.s Bálint, T. Grósz and V.l Chihaia Water-methanol Mixtures: Topology of Hydrogen Bonded Network Phys. Chem. Chem. Phys. 10, 5004 (2008)

33. J. W. Essex, W.L. Jorgensen, Dielectric constants of formamide and dimethylformamide via computer simulation J. Phys. Chem. 99, 17956, (1995) 34. W. L. Jorgensen and C. J. Swenson Optimized Intermolecular Potential Functions for Amides and Peptides. Hydration of Amides J. Am. Chem. SOC., 107, 1489 (1985)

35 J.M. M. Cordeiro $\mathrm{CH} . . . \mathrm{O}$ and $\mathrm{NH} . . . \mathrm{O}$ hydrogen bonds in liquid amides investigated by Monte Carlo simulation Int. J. Quantum Chemistry 65, 709, (1997

36. A. K. Soper, P. A.Egelstaff, Molec. Phys. 42, 399 (1981)

37 J. Z.Turner, A. K. Soper, J. L. Finney, Molec. Phys.70, 679 (1990)

38 I. P. Gibson, J. C. Dore, Molec. Phys. 37, 1281 (1979); H. Bertagnolli, P. Chieux, M. D. Zeidler, Molec.Phys. 32, 759 (1976)

39 R. L. McGreevy and L. Pusztai, Physica b. 234-236, 357 (1997)

40. L Pusztai, RL McGreevy, 'MCGR: an Inverse Method for Deriving the Pair Correlation Function', J. Neutron Res. 8, 17 (1999)

41. The MCGR software is avaibale on request from the authors. See ref. 40.

42. H. J. C. Berendsen, J. R. Grigera, T. P. Straatsma, J. Phys. Chem., 91, 6269 (1987)

43. M. Jelinska-Kazimierczuk and J. Szydlowski Physicochemical Properties of Solutions of Amides in $\mathrm{H} 2 \mathrm{O}$ and in D2O Journal of Solution Chemistry, . 30, 623 , (2001)

44. DL_POLY is a package of molecular simulation routines written by W. Smith and T. Forester, CCLRC, Daresbury Laboratory, Daresbury, Nr. Warrington, 1996. 45 R. Kumar, J. R. Schmidt, J. L. Skinner, J. Chem. Phys., 126, 204107 (2007) 
46. M. Matsumoto Relevance of hydrogen bond definitions in liquid water J. Chem. Phys 126, 054503 (2007)

47. R. H. Henchman and S. J. Irudayam Topological Hydrogen-Bond Definition to Characterize the Structure and Dynamics of Liquid Water J. Phys. Chem. B 114, 16792 (2010)

48. A. Geiger, F. Stillinger, A. Rahman,. J. Chem. Phys. 1979, 70, 4185. Aspects of the percolation process for hydrogen-bond networks in water J. Chem. Phys. 70, 4185 (1979)

49. L. B. Pártay and P. Jedlovszky I. Brovchenko and A. Oleinikova

Percolation Transition in Supercritical Water: A Monte Carlo Simulation Study J. Phys. Chem. B 111, 7603 (2007)

50. Pártay, L.; P.Jedlovszky, J. Line of percolation in supercritical water J. Chem. Phys. 123, $024502(2005)$

51 Stauffer, D. Introduction to Percolation Theory; Taylor and Francis: London, 1985.

52. N. Jan, Physica A, Large lattice random site percolation 266, 72 (1999)

53 Batagelj, A. Mrvar: Pajek - Program for Large Network Analysis. Home page http://vlado.fmf.uni-lj.si/pub/networks/pajek/.

54 V. Batagelj, A. Mrvar: Pajek - Analysis and Visualization of Large Networks. In Juenger, M., Mutzel, P. (Eds.): Graph Drawing Software. Springer (series Mathematics and Visualization), Berlin 2003. 77-103. ISBN 3-540-00881-0. • 77-103. ISBN 3-54000881-0.

55 V. Batagelj, A. Mrvar: Pajek - Program for Large Network Analysis. Connections 21 47. (1998)

56 .V. Chihaia, S. Adams, W. F. Kuhs, Chem. Phys., 2005, 317, 208-225. Molecular dynamics simulations of properties of a $\left(\begin{array}{lll}0 & 0 & 1\end{array}\right)$ methane clathrate hydrate surface Chem. Phys. 317, 208 (2005) 